\title{
Liver metastasis of colorectal cancer by protein-tyrosine phosphatase type 4A, 3 (PRL-3) is mediated through lymph node metastasis and elevated serum tumor markers such as CEA and CA19-9
}

\author{
KAZUHIKO HATATE, KEISHI YAMASHITA, KAZUYA HIRAI, HIROSHI KUMAMOTO, \\ TAKEO SATO, HEITA OZAWA, TAKATOSHI NAKAMURA, WATARU ONOZATO, \\ YUKIHITO KOKUBA, ATSUSHI IHARA and MASAHIKO WATANABE \\ Department of Surgery, Kitasato University, Kanagawa 228-8555, Japan
}

Received February 4, 2008; Accepted March 26, 2008

DOI: $10.3892 /$ or_00000068

\begin{abstract}
Phosphatase of regenerating liver (PRL)-3 was identified as a molecule associated with liver metastasis in colorectal cancer (CRC), although its precise causative role in distant metastasis remains elusive from a clinical point of view. The aim of this study was to promote the mechanistic insight of PRL-3 involvement in liver metastasis in CRC. One hundred and seven CRC patients with resection of the primary lesions were studied for clinicopathological and prognostic association with PRL-3 and were evaluated by immunohistochemistry in univariate and multivariate analyses. Intense immunostaining of PRL-3 was found in Dukes' A $(0 / 26)$, Dukes' B (0/30), Dukes' C (18/30) and Duke's D (20/21) although the PRL-3 expression could not predict metachronous liver metastasis (MLM) in Dukes' C patients. PRL-3 expression showed an inverse correlation of prognosis in a univariate prognostic analysis $(\mathrm{P}<0.0001)$, though a multivariate assay failed to demonstrate PRL-3 relevance as an independent prognostic factor. PRL-3 expression was closely associated with classic prognostic factors such as the $\mathrm{pN}$ factor $(\mathrm{P}<0.0001), \mathrm{H}$ factor-synchronous liver metastasis (SLM) $(\mathrm{P}<0.0001)$, pT factor $(\mathrm{P}=0.0002)$, preoperative CEA $(\mathrm{P}<0.0001)$ and preoperative CA19-9 $(\mathrm{P}<0.0001)$. Multivariate logistic regression analysis of PRL-3 expression revealed that the $\mathrm{pN}$ factor $(\mathrm{P}<0.0001)$, CEA $(\mathrm{P}<0.0001)$ and CA19-9 $(\mathrm{P}<0.0001)$ were finally remnant as an independent association with PRL-3. However, the H factor (SLM) was eliminated. Our data suggested that liver metastasis by PRL-3 is putatively mediated through lymph node metastasis and elevated tumor markers in the serum and the PRL-3 expression
\end{abstract}

Correspondence to: Professor Masahiko Watanabe, Department of Surgery, Medical School, Kitasato University, Kanagawa 228-8555, Japan

E-mail: gekaw@med.kitasato-u.ac.jp

Key words: liver metastasis, colorectal cancer, tyrosine-phosphatase, CEA, CA19-9 may not represent a direct causative mechanism of liver metastasis.

\section{Introduction}

Phosphatases and kinases are the cellular signal transduction enzymes that control protein phosphorylation. Protein tyrosine phosphatases (PTPs) play functional roles in regulating diverse proteins, which participate essentially in every aspect of cellular physiological and pathogenic processes (1). Phosphatase of regenerating liver (PRL)-1, -2 and -3 represents a novel class of PTPs superfamily members in possessing a unique $\mathrm{COOH}$-terminal prenylation motif with a PTP active site signature sequence CX5R (2). PRL phosphatases were found to be associated with the early endosome and plasma membrane in their prenylated state, while nuclear localization of these enzymes may occur in the absence of prenylation (3). Each of the proteins are of $\sim 20 \mathrm{kD}$ and share $75 \%$ amino acid sequence similarity. PRL-1 was originally identified as an immediate early gene whose expression was included in mitogen-stimulated cells and regenerating liver (4). Overexpression of PRL-1 and PRL-2 transformed mouse fibroblasts and hamster pancreatic epithelial cells in culture and promoted tumor growth in nude mice, suggesting that it may participate in tumorigenesis $(2,5)$.

Colorectal cancer (CRC) incidence and mortality has increased dramatically over the last decades in many regions and has become the second most common cancer worldwide. Metastasis of CRC in the liver is often responsible for the highest mortality rate and involvement of liver metastasis is an important prognostic factor for CRC. Mechanisms of liver metastasis of CRC are not fully clarified because of the involvement of multiple steps with the accumulation of an altered expression of a number of different genes. To gain insights into the molecular basis for metastasis, Saha et al compared the global gene expression profile of metastatic $\mathrm{CRC}$ with that of primary cancers, benign colorectal tumors, and normal colorectal epithelium (6). Overexpression of PRL-3 was elucidated to be associated with human CRC metastasis, and in 3 of 12 metastases examined, multiple copies of the PRL-3 gene were found within a small amplicon located at 
chromosome $8 \mathrm{q} 24.3(6,7)$. These data suggested that the PRL-3 gene is important for colorectal cancer metastasis and provide a new therapeutic target of these intractable lesions. However, the precise clinicopathological role of PRL-3 still remains elusive even in CRC from a prognostic point of view.

\section{Materials and methods}

Preparation of materials from patients with CRC. One hundred and seven Japanese CRCs were examined. Cancer patients underwent surgery at the Kitasato University Hospital. There were 64 male and 43 female patients with an average age of 64 and a range from 33 to 91 years. Among the 107 cases, 26, 30, 30 and 21 were classified as Dukes' stage A, B, C and D, respectively. The clinicopathological classifications are shown in Table I.

CRC was divided into either colon cancer or rectal cancer. Colon cancer consisted of cecal, ascending, transverse, descending, and sigmoid colon cancer. pT was designated as the following according to the 6th Japanese Classification of Colorectal Cancer (JCCC); pT0 (mucosal invasion), pT1 (submucosal invasion), pT2 (muscuralis propria invasion), pT3 (subserosal invasion or serosal exposure) and T4 (invasion to the surrounding organs). $\mathrm{pN}$ factor, $\mathrm{H}$ factor, LM factor and $\mathrm{P}$ factor represented lymph node metastasis, hepatic metastasis (synchronous liver metastasis: SLM), lung metastasis and peritoneal dissemination, respectively.

Immunohistochemical study. We examined PRL-3 immunostaining in 5 liver metastatic lesions of CRC and 5 normal colorectal mucosal tissues in order to confirm a positive and negative control of PRL-3, respectively, with 4 kinds of antibody \#1 anti-human/-mouse/-rat PRL-3 antibody Clone 334402 (mouse monoclonal $\mathrm{IgG}_{2 \mathrm{~B}}$, R\&D Systems Inc., Minneapolis, MN), \#2 anti-PRL-3 antibody GB63023 (rabbit monoclonal, Genesis Biotech Inc., Taipei, Taiwan), \#3 antihuman/-mouse PRL-3 antibody (mouse monoclonal $\mathrm{IgG}_{1}$, Abgent Inc., San Diego, CA) and \#4 anti-PRL-3 antibody (rabbit polyclonal, Zymed Laboratories Inc., South San Francisco, CA). Among them, \#1 showed much more remarkable contrasts between the positive and negative controls when an antibody was 20-fold diluted (in a comparison with 50- and 100-fold dilution of \#1 or other antibodies). Under the best optimized conditions, 5 liver metastatic lesions of CRC and 5 normal colorectal mucosal tissues were all positive and negative, respectively. We then applied this immunohistochemical technique to 107 paraffinembedded CRC. We scored the expression as negative when $<10 \%$ of the carcinoma cells were stained in an examined area of a specimen.

\section{Results}

PRL-3 immunostaining identified in CRC and univariate clinicopathological analysis. Intense PRL-3 immunostaining was identified in 38 cases out of 107 CRC patients (36.4\%), while weak staining and none to faint staining were found in $15(14.0 \%)$ and 54 cases (50.5\%), respectively (Fig. 1 and Table I). A clinicopathological analysis was performed for basic clinical factors according to PRL-3 immunostaining status (Table I) and revealed that PRL-3 expression is significantly involved in classic prognostic factors such as $\mathrm{pT}$ $(\mathrm{P}=0.0002), \mathrm{pN}(\mathrm{P}<0.0001)$, histology $(\mathrm{P}=0.0002), \mathrm{P}$ factor $(\mathrm{P}=0.06)$, preoperative $\mathrm{CEA}(\mathrm{P}<0.0001)$, preoperative CA19-9 $(\mathrm{P}<0.0001)$ and Dukes stage $(\mathrm{P}<0.0001)$.

We performed a univariate prognostic analysis using the log-rank test and revealed that a poor prognosis of CRC patients was significantly associated with $\mathrm{pN}$ factor $(\mathrm{P}<0.0001)$, histology $(\mathrm{P}=0.019), \mathrm{H}$ factor $(\mathrm{SLM})(\mathrm{P}<0.0001)$, LM factor $(\mathrm{P}=0.035)$, $\mathrm{P}$ factor $(\mathrm{P}<0.0001)$, preoperative serum CEA value $(\mathrm{P}<0.0001)$ and preoperative serum $C A 19-9$ value $(\mathrm{P}=0.0007)$. We could not assess the prognostic relevance according to pT and Dukes staging using StatView 5.0 software, since there was no censored cases with T0, 1, 2 and Dukes A CRC patients among cases we examined. For the total 107 CRC, intense immunostaining of PRL-3 showed a remarkable prognostic difference as compared with none to weak immunostaining of PRL-3 ( $\mathrm{P}<0.0001$, Fig. 2A).

Multivariate prognostic analysis revealed that $P R L-3$ involvement in SLM may be mediated by lymph node metastasis and elevated tumor markers. In the current study, we could not assess the prognostic relevance according to $\mathrm{pT}$ factor as stated earlier and our multivariate hazard proportional model was restricted to the 85 pT3,4 CRC, which showed that PRL-3 could not be an independent prognostic factor and that finally remnant independent prognostic factors were $\mathrm{H}$ factor $(\mathrm{SLM})(\mathrm{HR}=10.0$; $\mathrm{p}<0.0001)$, preoperative CA19-9 $(\mathrm{HR}=3.89 ; \mathrm{p}=0.01), \mathrm{pN}$ $(\mathrm{HR}=3.70 ; \mathrm{p}=0.028)$ and preoperative CEA $(\mathrm{HR}=2.73$; $\mathrm{p}=0.02$ ) (Table II). To reveal the reason for elimination of PRL-3 expression as an independent prognostic factor, we performed a multivariate logistic regression analysis as to PRL-3 immunostaining for each of the univariate prognostic factors with $\mathrm{p}<0.1$ and revealed that PRL-3 expression was independently associated with lymph node metastasis (RR: relative risk $=5.78 ; \mathrm{p}<0.0001)$, preoperative CEA $(\mathrm{RR}=5.27$; $\mathrm{p}<0.0001)$ and preoperative CA19-9 $(\mathrm{RR}=3.93$; $\mathrm{p}<0.0001)$, while $\mathrm{H}$ factor was eliminated as an independent association with PRL-3 expression. The multivariate analyses led us to speculate that PRL-3 involvement in SLM may be mediated by lymph node metastasis and elevated tumor markers (Fig. 3).

Relationship of PRL-3 immunostaining to metachronous liver metastasis (MLM) and prognosis with Dukes C CRC patients. We then examined the relationship between PRL-3 immunostaining and MLM using Dukes C CRC patients, since MLM occurred largely among Dukes $C$ patients and rarely among Dukes B and A patients. Among 30 Dukes C CRC patients examined in this current study, MLM was found in 16 cases (53\%) during a follow-up course. A PRL-3 immunostaining pattern did not have any value in predicting MLM in Dukes C CRC patients as follows: the patterns subdivided into intense/weak/none to faint immunostaining of PRL-3 in absence and presence of MLM are 9/2/3 cases and $9 / 4 / 3$ cases, respectively (no significant difference). As reflected this finding, PRL-3 immunostaining did not have any prognostic value if restricted to Dukes C CRC patients (Fig. 2B). These findings may indicate that PRL-3 expression 


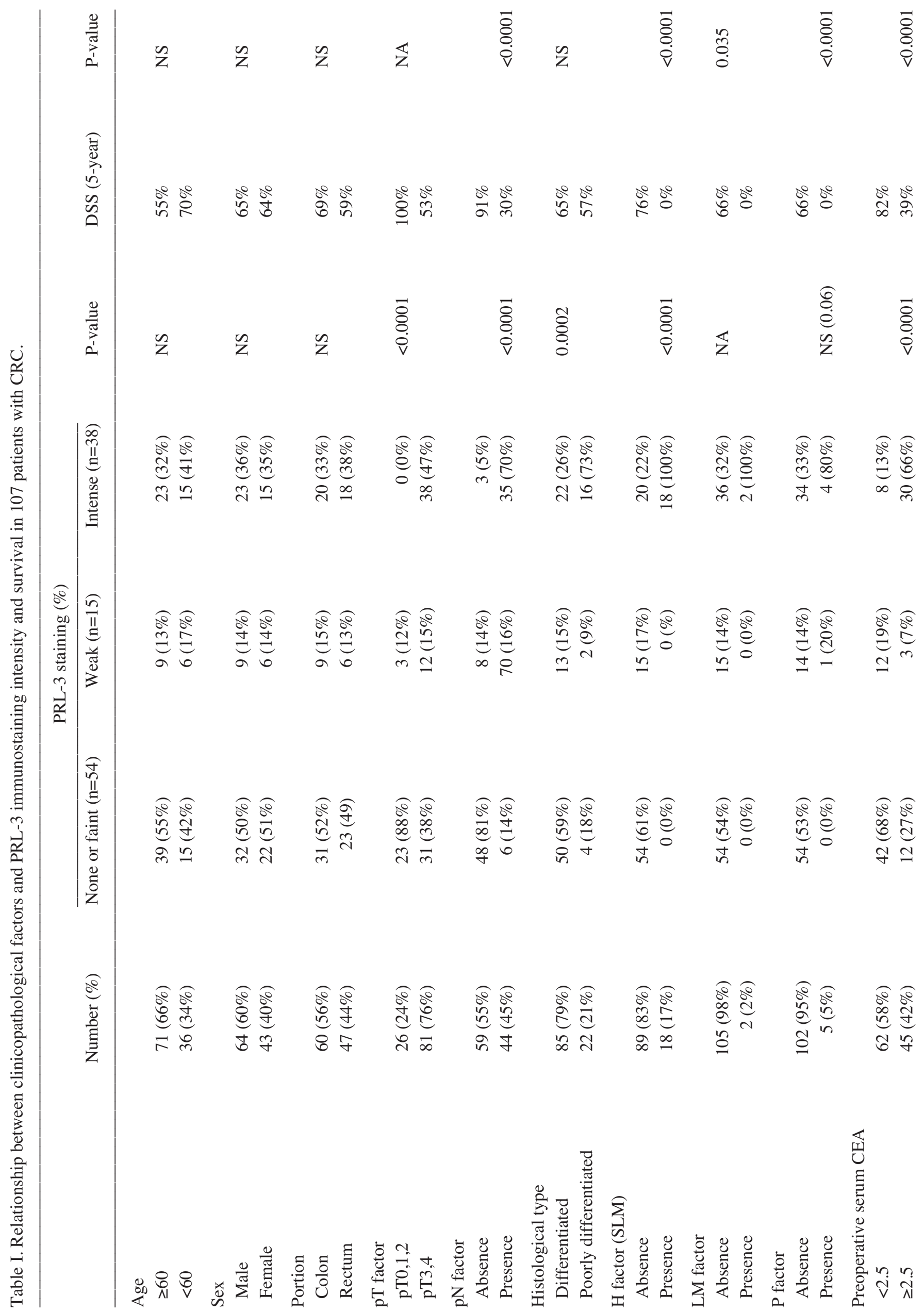


is not directly involved in liver metastasis, even though it is either synchronous or metachronous.

\section{Discussion}

In this study, PRL-3 is more closely associated with lymph node metastasis than SLM and univariate PRL-3 association with SLM is deemed just to reflect unique biological characters of CRC. PRL-3 is independently associated with lymph node metastasis, CEA and CA19-9 (Table III), which were in turn an independent prediction of SLM by a multivariate logistic regression analysis (data not shown). A large portion of SLM occurs in patients with lymph node metastasis (18 out of 21 Dukes D cases). These findings indicated that PRL-3 is involved in SLM through lymph node metastasis and elevated tumor markers. Kato et al proposed PRL-3 as being a potential for the prediction of MLM in CRC (8). However, the prediction value becomes effective for clinical use if its value is restricted to Dukes $\mathrm{C}$ patients, since MLM is very rare in Dukes A and B CRC patients. As our data are based on a small number of patients in Dukes $\mathrm{C}$ $(n=30)$, we did not expect a good potential of PRL-3 expression predicting MLM (Fig. 2B). Taking the two negative aspects of SLM and MLM as to PRL-3 expression into account, we had to conclude that PRL-3 expression is significantly involved in metastatic potential of lymph nodes, though not directly in liver metastasis.

The Vogelstein lab initially identified PRL-3 as a consistent association with liver metastasis $(6,7)$, where they reported that PRL-3 expression is found in all the lymph node metastasis of CRC by in situ hybridization $(\mathrm{n}=4)(7)$. This finding was validated by a recent immunohistochemical analysis in CRC (9). Furthermore, reports that PRL-3 could reflect lymph node metastasis have been increasing even in other organs such as gastric cancer $(10,11)$ and breast cancer (12). In gastric cancer, PRL-3 expression was detected in 68\% of primary lesions (with nodal metastasis $81.5 \%$, without nodal metastasis 50\%; $\mathrm{p}=0.004)$ and the incidence of PRL-3 expression in lymph node metastasis was significantly higher than that in primary gastric cancers $(\mathrm{p}<0.001)$. Moreover, PRL-3 expression was closely associated with lymphatic invasion ( $\mathrm{p}=0.002)$, extent of lymph node metastasis $(\mathrm{p}=0.002)$ and tumor stage $(\mathrm{p}=0.045)$, which suggested that PRL-3 expression may play a significant role in the invasion and metastasis of gastric carcinoma. Lymph node metastasis is one of the major concerns in the cancer research field, since the metastasis to lymph nodes is essential for cancer development. Several microarray studies profiling the expression of cancer tissues remains unresolved for bona fide therapeutic target molecules involved in lymph node metastasis $(13,14)$. Hence we are now proposing that PRL-3 may surrogate the lymph node metastatic phenotype for molecular insight or tumor maker development. A PRL-3 knock-down may elucidate an important mechanism of lymph node metastasis or identify markers of lymph node diseases.

Notably, alterations of CA19-9 and CEA in the preoperative serum were closely associated with PRL-3 expression status independently of lymph node metastasis (Fig. 3). This is the first report in which an oncogenic molecule was associated with the serum level of CEA and CA19-9. It 


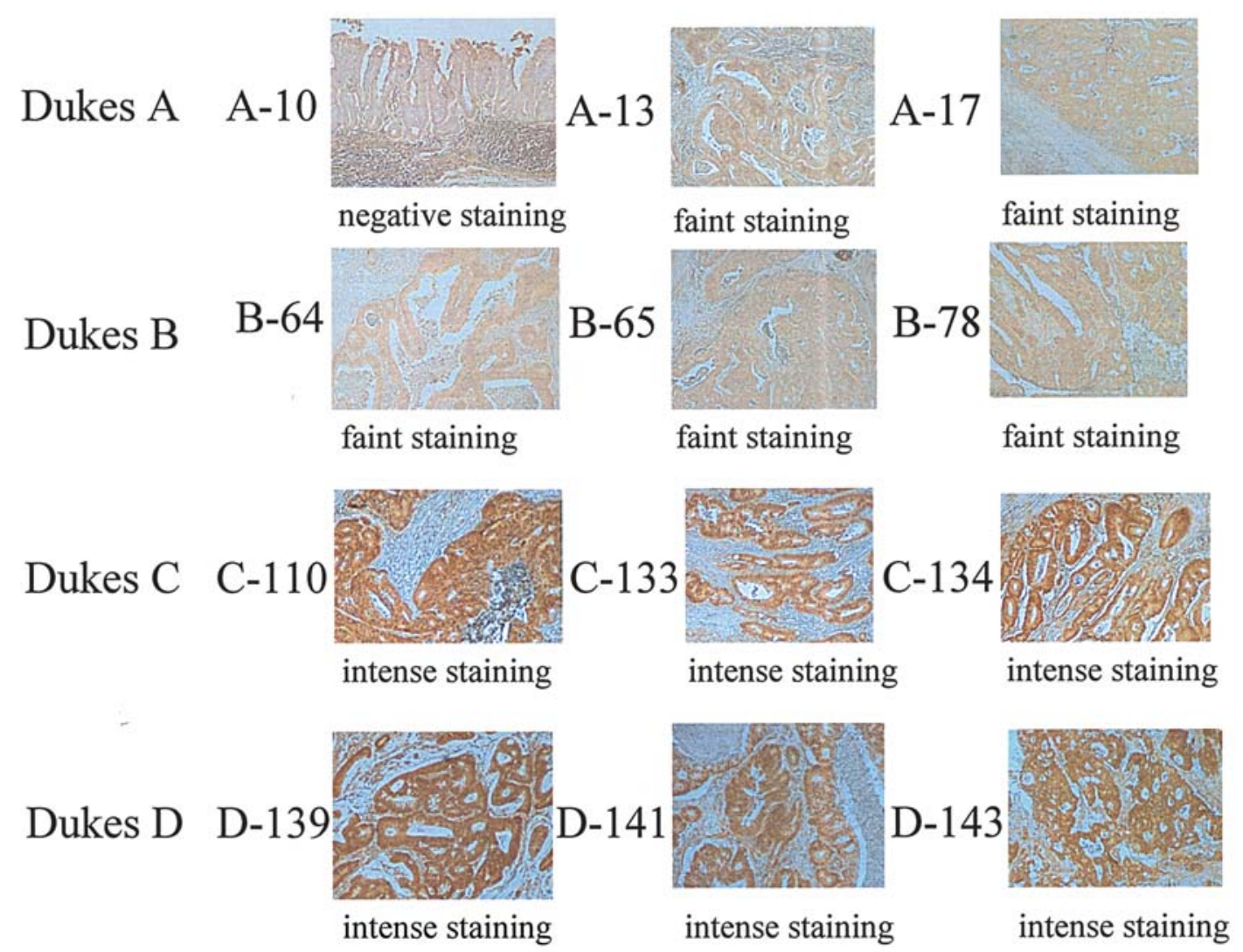

Figure 1. PRL-3 immunostaining of representative CRC cases. Every 3 cases are shown in Dukes A, B, C and D stages. Patterns of staining are depicted below each picture as negative, faint or intense staining of PRL-3.

Table II. A multivariate analysis of factors associated with disease-specific survival.

\begin{tabular}{lccc}
\hline & \multicolumn{3}{c}{ Tumor factor } \\
\cline { 2 - 4 } Variable & HR & $95 \%$ CI & P-value \\
\hline H factor (SLM) & 10 & $3.37-25.00$ & $<0.0001$ \\
Preoperative CA19-9 & 3.89 & $1.35-11.19$ & 0.01 \\
pN factor & 3.7 & $1.15-11.90$ & 0.028 \\
Preoperative CEA & 2.73 & $1.17-06.41$ & 0.02 \\
P factor & 3.47 & $0.79-15.38$ & $\mathrm{NS}$ \\
PRL-3 & 2.06 & $0.68-06.21$ & $\mathrm{NS}$ \\
LM & 1.46 & $0.32-06.66$ & $\mathrm{NS}$ \\
\hline
\end{tabular}

$\mathrm{HR}$, hazard ratio; $\mathrm{CI}$, confidence interval; $\mathrm{P}$, peritoneal dissemination and NS, not significant.

is worth noting that cases with all the 3 positive parameters were only found in CRC with intense PRL-3 (see green box in Fig. 3) $(\mathrm{p}<0.0001)$. Cases with at least 2 positive parameters were recognized in 30 (79\%) out of 38 intense PRL-3 cases, while in $2(13 \%)$ out of 15 weak PRL-3 cases and 3 out of 54 none to faint PRL-3 cases $(6 \%)(\mathrm{p}<0.0001)$ (see blue green box in Fig. 3). These findings suggested that PRL-3 affects positivity of at least 2 factors among the three independent prognostic factors (lymph node metastasis, preoperative CEA and preoperative CA19-9) (Table II).
Table III. Factors associated with PRL3 immunostaining on multivariate logistic-regression analysis.

\begin{tabular}{lcc}
\hline & RR & P-value \\
\hline $\mathrm{pN}$ & 5.78 & $<0.0001$ \\
Preoperative CEA & 5.27 & $<0.0001$ \\
Preoperative CA19-9 & 3.93 & $<0.0001$ \\
P factor & 1.83 & $\mathrm{NS}$ \\
PT & 0.045 & $\mathrm{NS}$ \\
H factor (SLM) & 0.041 & $\mathrm{NS}$ \\
LM & 0.034 & $\mathrm{NS}$ \\
\hline
\end{tabular}

$\mathrm{RR}$, relative risk.

Prognostic value of serum CEA or CA19-9 has become evident, while it remains controversial whether it is causative or results in tumor progression (15). Previous basic research supported a novel notion of tumor-induced immune suppression and has proven to be at least partially mediated by CEA and CA19-9. CEA molecules which shed from the cancer cell surface are phagocytosed by the macrophage in the liver and activate for the release of various cytokines so as to make the environment suitable for the implantation of metastatic tumors $(16,17)$. However, CA19-9 is a glycoform epitope, which is supposed to be abundantly expressed on the surface of O-type mucin such as MUC1 and is supposed to 
A

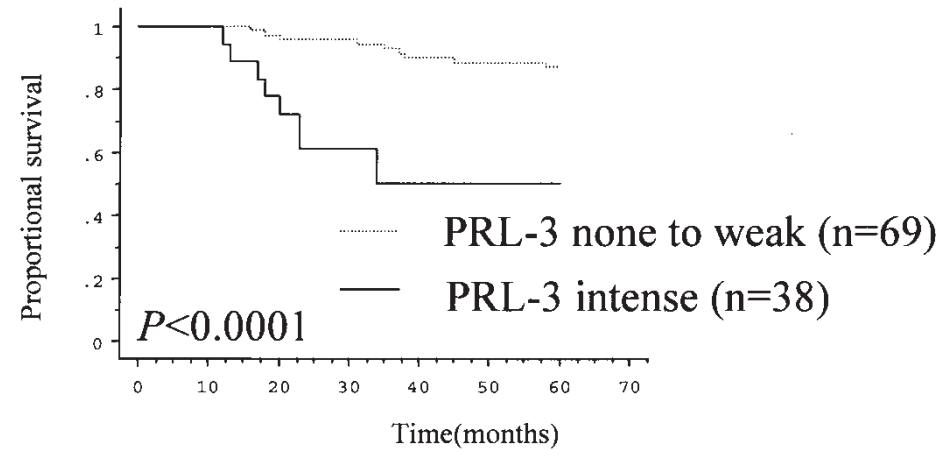

B

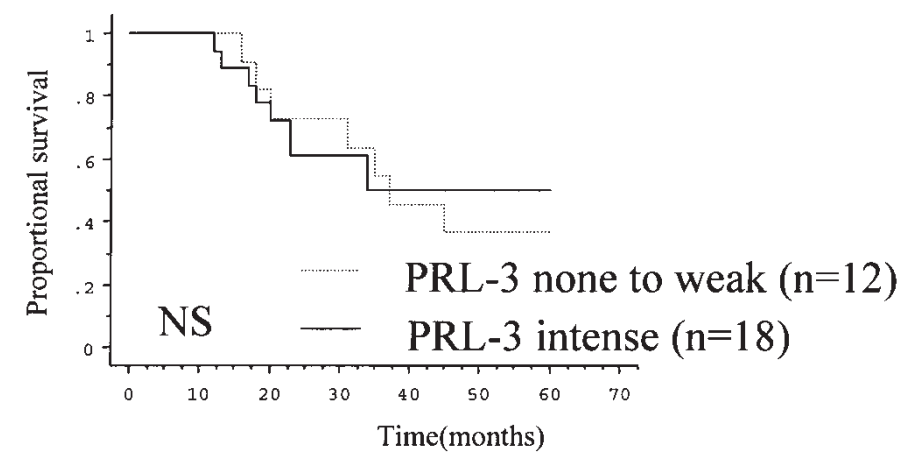

Figure 2. Disease-specific survival by Kaplan-Meier analysis. (A) Total cases of CRC were analyzed according to PRL-3 expression. Patients with intense staining of PRL-3 showed a significantly poorer prognosis than those with none to weak staining of PRL-3 ( $\mathrm{P}<0.0001)$. (B) Patients with intense staining of PRL-3 did not show a significantly poorer prognosis than those with none to weak staining of PRL-3 in 30 Dukes C CRC cases (not statistically significant).

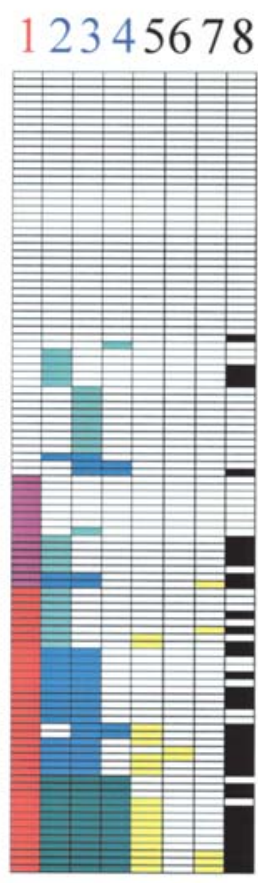

1 PRL-3

2 Lymph node metastasis

3 Preoperative CEA

4 Preoperative CA19-9

5 H factor

$6 \mathrm{LM}$ factor

7 P factor

8 Death due to recurrence

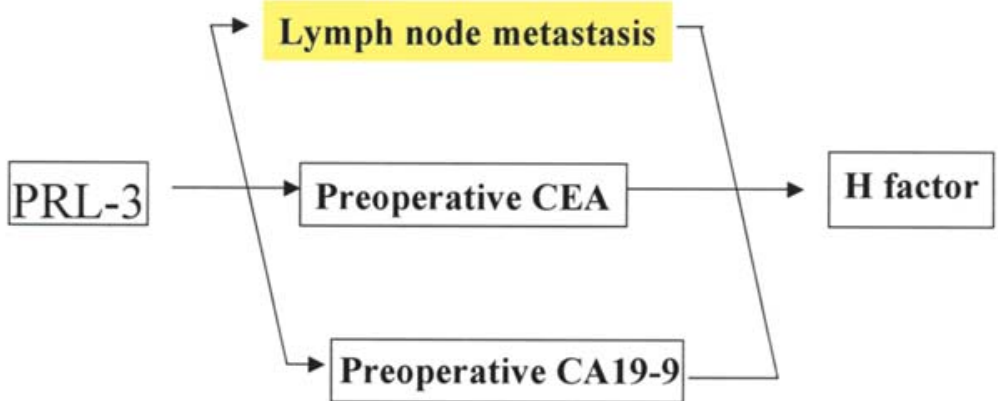

Figure 3. PRL-3 relationship with its independent predictors. A multivariate logistic regression analysis revealed that PRL-3 expression is independently associated with lymph node metastasis, preoperative CEA and preoperative CA19-9. Red box, intense immunostaining of PRL-3; pink box, weak immunostaining of PRL-3; white box in line 1, none to faint immunostaining of PRL-3; green box, 3 positive cases of the independent predictors of PRL-3; green blue box, 2 positive cases of the independent predictors of PRL-3; thin green box, 1 positive case of the independent predictors of PRL-3; white box in line 2 to 4 ; no positive case of the 3 independent predictors of PRL-3. 
be evident after transformation (18). Such malignant mucin could induce COX2/PGE2 production in macrophages in the tissues (19) or even in the blood (20) and therefore serum CA19-9 is associated with serum PGE2 in gastrointestinal cancer (20). It appears that COX-2/PGE2 induced via mucins represented by the CA19-9 epitope could be causative for tumor-induced immune suppression. These findings suggested that elevated tumor markers associated with PRL-3 may involve metastatic disease development of CRC through immune suppression.

PRL phosphatases constitute a novel class of small (20 kDa), prenylated phosphatase with oncogenic activity and represent a new therapeutic target. For angiogenic or metastatic ability, a causative role of PRL-3 phosphatase activity was confirmed (21-23). Hence, the catalytic domain of PRL-3 could serve as an ideal therapeutic target for drug development in blocking the spread of CRC. Kozlov et al presented the solution structure of PRL-3, the first structure of a PRL-3 phosphatase and identified a potential regulatory role of Cys (49) that forms an intramolecular disulfide bond with the catalytic Cys (104), even under mildly reducing conditions (24). Recently, the first report on PRL-3 inhibitors was very promising for the target therapy of CRC, isolated from natural sources as two biflavonoids, ginkgetin and sciadopitysin, from the $\mathrm{MeOH}$ extract of the young branches of Taxus cuspidate, which inhibited phosphatase of regenerating liver-3 (PRL-3) with $\mathrm{IC}_{50}$ values of 25.8 and $46.2 \mu \mathrm{M}$, respectively (25).

In conclusion, our current research identified four independent prognostic factors, SLM-H factor, preoperative CEA, preoperative CA19-9 and lymph node metastasis (Table II) and elucidated the relationship between PRL-3 expression and these prognostic factors. Our conclusion is that PRL-3 is associated with SLM, however, liver metastasis by PRL-3 is mediated by lymph node metastasis and elevated tumor markers and thereafter we interpret that lymph node metastasis and tumor-induced immune suppression may be needed for distant metastasis. Such a relevant relationship of prognostic factors may be a clue for an actual therapeutic strategy and PRL-3 inhibition could be promising in CRC.

\section{References}

1. Zhang ZY, Zhou B and Xie L: Modulation of protein kinase signaling by protein phosphatases and inhibitors. Pharmacol Ther 93: 307-317, 2002.

2. Cates CA, Michael RL, Stayrook KR, Harvey KA, Burke YD, Randall SK and Crowell PL: Prenylation of oncogenic human PTPcaax protein tyrosine phosphatases. Cancer Lett 110: 49-55, 1996.

3. Zeng Q, Si X, Horstmann H, Xu Y, Hong W and Pallen CJ: Prenylation-dependent association of protein-tyrosine phosphatases PRL-1, -2 , and -3 with the plasma membrane and the early endosome. J Biol Chem 275: 21444-21452, 2000.

4. Mohn KL, Laz TM, Hsu JC, Melby AE, Bravo R and Taub R: The immediate-early growth response in regenerating liver and insulin-stimulated H-35 cells: comparison with serum-stimulated $3 \mathrm{~T} 3$ cells and identification of 41 novel immediate-early genes. Mol Cell Biol 11: 381-390, 1991.

5. Diamond RH, Cressman DE, Laz TM, Abrams CS and Taub R: PRL-1, a unique nuclear protein tyrpsine phosphatase, affects cell growth. Mol Cell Biol 14: 3752-3762, 1994.

6. Saha S, Bardelli A, Buckhaults P, Velculescu VE, Rago C, St Croix B, Romans KE, Choti MA, Lengauer C, Kinzler KW and Vogelstein B: A phosphatase associated with metastasis of colorectal cancer. Science 294: 1343-1346, 2001.
7. Bardelli A, Saha S, Sager JA, Romans KE, Xin B, Markowitz SD, Lengauer C, Velculescu VE, Kinzler KW and Vogelstein B: PRL-3 expression in metastatic cancers. Clin Cancer Res 9: 5607-5615, 2003.

8. Kato H, Semba S, Miskad UA, Seo Y, Kasuga M and Yokozaki H: High expression of PRL-3 promotes cancer cell motility and liver metastasis in human colorectal cancer: a predictive molecular marker of metachronous liver and lung metastases. Clin Cancer Res 10: 7318-7328, 2004.

9. Wang Y, Li ZF, He J, Li YL, Zhu GB, Zhang LH and Li YL: Expression of the human phosphatases of regenerating liver (PRLs) in colonic adenocarcinoma and its correlation with lymph node metastasis. Int J Colorectal Dis 22: 1179-1184, 2007.

10. Miskad UA, Semba S, Kato H and Yokozaki H: Expression of PRL-3 phosphatase in human gastric carcinoma: close correlation with invasion and metastasis. Pathology 71: 176-184, 2004.

11. Miskad UA, Semba S, Kato H, Matsukawa Y, Kodama Y, Mizuuchi E, Maeda N, Yanagihara K and Yokozaki H: High PRL-3 expression in human gastric cancer is a marker of metastasis and grades of malignancies: an in situ hybridization study. Virchows Arch 450: 303-310, 2007.

12. Radke I, Gotte M, Kersting C, Mattsson B, Kiesel L and Wülfing P: Expression and prognostic impact of the protein tyrosine phosphatases PRL-1, PRL-2, and PRL-3 in breast cancer. Br J Cancer 95: 347-354, 2006.

13. Inoue H, Matsuyama A, Mimori K, Ueo H and Mori M: Prognostic score of gastric cancer determined by cDNA microarray. Clin Cancer Res 8: 3475-3479, 2002.

14. Jinawath N, Furukawa Y, Hasegawa S, Li M, Tsunoda T, Satoh S, Yamaguchi $\mathrm{T}$, Imamura $\mathrm{H}$, Inoue $\mathrm{M}$, Shiozaki $\mathrm{H}$ and Nakamura Y: Comparison of gene-expression profiles between diffuse- and intestinal-type gastric cancers using a genome-wide cDNA microarray. Oncogene 23: 6830-6844, 2004.

15. Locker GY, Hammilton S, Harris J, Jessup JM, Kemeny N, Macdonald JS, Somerfield MR, Hayes DF and Bast RC Jr: ASCO 2006 update of recommendation for the use of tumor markers in gastrointestinal cancer. J Clin Oncol 24: 5313-5327, 2006.

16. Edmiston KH, Gangopadhyay A, Shoji Y, Nachman AP, Thomas P and Jessup JM: In vivo induction of murine cytokine production by carcinoembryonic antigen. Cancer Res 57: 4432-4436, 1997.

17. Gangopadhyay A, Lazure DA and Thomas P: Adhesion of colorectal carcinoma cells to the endothelium is mediated by cytokines from CEA stimulated Kupffer cells. Clin Exp Metastasis 16: 703-712, 1998.

18. Miyazaki K, Ohmori K, Izawa M, Koike T, Kumamoto K, Furukawa K, Ando T, Kiso M, Yamaji T, Hashimoto Y, Suzuki A, Yoshida A, Takeuchi M and Kannagi R: Loss of disialyl Lewis(a), the ligand for lymphocyte inhibitory receptor sialic acid-binding immunoglobulin-like lectin-7 (Siglec-7) associated with increased sialyl Lewis(a) expression on human colon cancers. Cancer Res 64: 4498-4505, 2004.

19. Inaba T, Sano H, Kawahito Y, Hla T, Akita K, Toda M, Yamashina I, Inoue $\mathrm{M}$ and Nakada H: Induction of cyclooxygenase-2 in monocyte/macrophage by mucins secreted from colon cancer cells. Proc Natl Acad Sci USA 100: 2736-2741, 2003.

20. Yokoigawa N, Takeuchi N, Toda M, Inoue M, Kaibori M, Yanagida H, Inaba T, Tanaka H, Ogura T, Takada H, Okumura T, Kwon AH, Kamiyama Y and Nakada H: Overproduction of PGE2 in peripheral blood monocytes of gastrointestinal cancer patients with mucins in their bloodstream. Cancer Lett 245: 149-155, 2007.

21. Zeng Q, Dong JM, Guo K, Li J, Tan HX, Koh V, Pallen CJ, Manser E and Hang W: PRL-3 and PRL-1 promote cell migration, invasion, and metastasis. Cancer Res 63: 2716-2722, 2003.

22. Wu X, Zeng H, Zhang X, Zhao Y, Sha H, Ge X, Zhang M, Gao X and $\mathrm{Xu} \mathrm{O}$ : Phosphatase of regenerating liver-3 promotes motility and metastasis of mouse melanoma cells. Am J Pathol 164: 2039-2054, 2004.

23. Guo K, Li J, Tang JP, Koh V, Gan BQ and Zeng Q: Catalytic domain of PRL-3 plays an essential role in tumor metastasis: formation of PRL-3 tumors inside the blood vessels. Cancer Biol Ther 3: 945-951, 2004.

24. Kozlov G, Cheng J, Ziomek E, Banville D, Gehring K and Ekiel I: Structural insights into molecular function of the metastasisassociated phosphatase PRL-3. J Biol Chem 279: 11882-11889, 2004

25. Choi SK, Oh HM, Lee SK, Jeong DG, Ryu SE, Son KH, Han DC, Sung ND, Baek NI and Kwon BM: Biflavonoids inhibited phosphatase of regenerating liver-3 (PRL-3). Nat Prod Res 20: 341-346, 2006. 